\title{
Magnetic-texture-controlled transverse spin injection
}

\author{
E. van der Bijl, R. E. Troncoso, and R. A. Duine \\ Institute for Theoretical Physics, Utrecht University, Leuvenlaan 4, 3584 CE Utrecht, The Netherlands
}

(Received 7 January 2013; revised manuscript received 12 April 2013; published 21 August 2013)

\begin{abstract}
We propose an effect whereby an electric current along the interface between a ferromagnetic and normal metal leads to injection of pure spin current into the normal metal, if the magnetization direction in the ferromagnet varies spatially along the direction of current. For the specific example of a ferromagnetic spin spiral, we compute the inverse spin Hall effect voltage this spin current gives rise to when injected into a Pt layer. Furthermore, we show that this pure spin current leads to modification of the parameters that govern spin transfer and current-driven domain-wall motion, which can be used to tune the latter in layered magnetic systems. This effect in principle enables in situ control over the location, magnitude, and polarization of spin-current injection in devices.
\end{abstract}

DOI: 10.1103/PhysRevB.88.064417

PACS number(s): 75.70. $-\mathrm{i}, 75.60 . \mathrm{Ch}, 75.76 .+\mathrm{j}$

\section{INTRODUCTION}

Spintronic devices make use of the spin degree of freedom to process and store information. Hence, the generation and detection of nonequilibrium spin accumulation and spin currents is of paramount importance. In particular, all-electric injection and control of spin currents at room temperature and without high magnetic fields is crucial for viable integration with and as extension of current technology. ${ }^{1}$ To obtain spin currents, a large variety of physical mechanisms and geometries are investigated. ${ }^{2,3}$ One class of approaches relies on parametric pumping. ${ }^{4}$ In these, a periodic (ac) excitation is transformed into a dc spin current. Examples are circularly polarized optical photons,,${ }^{3,5-7}$ magnons, ${ }^{8-10}$ acoustic waves, ${ }^{11}$ and single-domain ferromagnetic resonance. ${ }^{12-14}$ For example, recently the injection of pure spin current using this last method was demonstrated for $p$-type $\mathrm{Si}^{15}$

In contrast to the pumping approaches described above, a spin accumulation can also be obtained via a static bias. A current through a ferromagnet-nonmagnetic metal (FM-NM) junction causes spin injection into the nonmagnetic layer. ${ }^{16-18}$ Room-temperature injection in silicon was demonstrated in Ref. 19. Spin-orbit coupling also opens the possibility to create spin currents using electric fields only. For example, the spin Hall effect generates a spin current transverse to a charge current. ${ }^{20-23}$

The interplay between heat flow and spin currents studied in the young field of spin caloritronics yields novel ways to inject pure spin currents using temperature gradients. For example, heat flow leads to the generation of spin accumulation via the spin Seebeck effect, ${ }^{24}$ spin-dependent Seebeck effect, ${ }^{25}$ and spin Seebeck tunneling. ${ }^{26}$ Controlling spin currents with charge flow is the prime occupation of spintronics. Research on spin currents, however, has impact beyond this field, and has led, for example, to the discovery of time-reversal-invariant topological insulators. ${ }^{27}$

In this article we propose a mechanism for the injection of a spin current into the normal metal in a FM-NM heterostructure that is perpendicular to the FM-NM interface and transverse to the electric current that flows along the interface (see Fig. 1). Consider a static magnetic texture in the FM layer such that the direction of magnetization $\boldsymbol{m}(\boldsymbol{x})$ depends on the coordinate along the wire, and such that electric current flows along the wire. We denote the charge current density on the interface by $\boldsymbol{j}_{c}$. It is the magnitude of this charge current density that governs the magnitude of the injected spin current. As we discuss in detail below, the change in orientation of the magnetization due to the presence of a magnetization gradient will cause a transverse spin current $\boldsymbol{j}_{s}^{\text {in }}$ into the NM given by

$$
\boldsymbol{j}_{s}^{\text {in }}(\boldsymbol{x})=\frac{\hbar g}{4 \pi G_{0}} \boldsymbol{m}(\boldsymbol{x}) \times\left[\left(\boldsymbol{v}_{s} \cdot \nabla\right) \boldsymbol{m}(\boldsymbol{x})\right],
$$

where $\boldsymbol{j}_{s}^{\text {in }}(\boldsymbol{x})$ is the injected spin-current density flowing perpendicular to the interface (and hence transverse to the charge current direction) with spin polarization in the direction of the helicity $\boldsymbol{m} \times\left(\boldsymbol{j}_{c} \cdot \nabla\right) \boldsymbol{m}$ of the magnetization texture along the current direction (in the $x$ direction for the situation in Fig. 1). Here, $\boldsymbol{m}(\boldsymbol{x})$ is the unit-vector magnetization direction of the FM layer, $\boldsymbol{v}_{s}=-g_{L} \mu_{B} P \boldsymbol{j}_{c} / 2 M_{s}|e|$ is the spin velocity, $P$ is the spin polarization of the current density at the interface, $g_{L}$ is the Landé $g$ factor, $\mu_{B}$ is the Bohr magneton, $M_{S}$ is the saturation magnetization, and $-|e|$ is the charge of an electron. Moreover, $G_{0}=2 e^{2} / h$ is the quantum of conductance. Furthermore, the parameter $g$ is governed by the spin-dependent transport properties of the FM-NM interface. Equation (1) is the main equation describing the effect we propose in this paper.

In the next section we give a scattering-theory expression for the interface parameter $g$, that can be evaluated using $a b$ initio methods. The scattering theory is valid for treating the interface which is atomically sharp, and yields boundary conditions for the diffusive description appropriate for the bulk.

\section{SCATTERING THEORY}

We consider a two-dimensional tight-binding model for the interface between ferromagnet and normal metal. The ferromagnetism is described with localized magnetic moments exchange coupled to the electrons, and connected on every site $\mathbf{r}=\left\{i_{x}, i_{y}\right\}$ to a metallic reservoir (i.e., the normal metal) with chemical potentials $\mu_{\mathbf{r}}$. The system is described by the Hamiltonian $\mathcal{H}=\mathcal{H}_{S}+\mathcal{H}_{L}+\mathcal{H}_{C}$ representing the electronic system in the FM, NM leads and contacts between them, respectively. The first term is specified in terms of second-quantized operators $\hat{c}_{\mathbf{r}, \sigma}\left(\hat{c}_{\mathbf{r}, \sigma}^{\dagger}\right)$ that annihilate (create) 


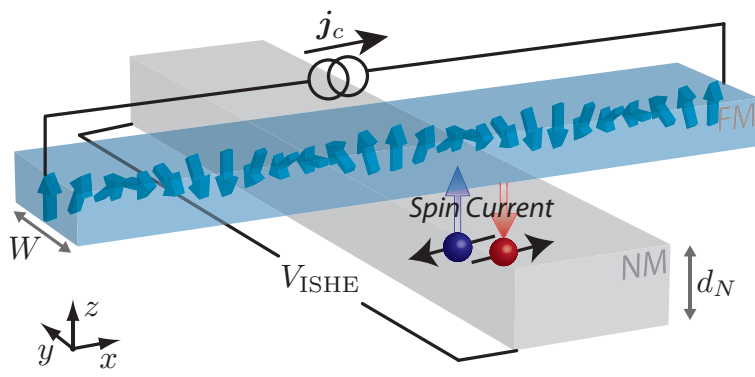

FIG. 1. (Color online) A charge current along the interface between a ferromagnet containing a magnetic texture and a normal metal leads to the injection of a transverse pure spin current at the interface. The direction of the spin component of the injected spin current is in the direction of the helicity $\boldsymbol{m} \times\left(\boldsymbol{j}_{c} \cdot \nabla\right) \boldsymbol{m}$ of the spin spiral along the current direction, leading to a spin accumulation in the normal metal.

an electron with spin $\sigma$ at site $\mathbf{r}$ :

$$
\mathcal{H}_{S}=-J_{S} \sum_{\left\langle\mathbf{r}, \mathbf{r}^{\prime}\right\rangle ; \sigma} \hat{c}_{\mathbf{r}, \sigma}^{\dagger} \hat{c}_{\mathbf{r}^{\prime}, \sigma}-\Delta \sum_{\mathbf{r} ; \sigma, \sigma^{\prime}} \hat{c}_{\mathbf{r}, \sigma}^{\dagger} \boldsymbol{m}_{\mathbf{r}} \cdot \boldsymbol{\tau}_{\sigma, \sigma^{\prime}} \hat{c}_{\mathbf{r}, \sigma^{\prime}}
$$

which describes nearest-neighbor (indicated by the brackets $\langle\cdot, \cdot\rangle)$ hopping with amplitude $J_{S}$ and coupling to the local magnetic moments $\boldsymbol{m}_{\mathbf{r}}$, where $\Delta$ is the exchange energy and $\boldsymbol{\tau}$ is the Pauli spin-matrix vector. The metallic contact is described by a set of one-dimensional leads at chemical potential $\mu_{\mathbf{r}}$ and modeled by the Hamiltonian $\mathcal{H}_{L}=\sum_{\mathbf{r}} \mathcal{H}_{\mathbf{r}}$ where the Hamiltonian for the lead connected to site $\mathbf{r}$ is given by $\mathcal{H}_{\mathbf{r}}=-J_{L_{\mathbf{r}}} \sum_{\left\langle j^{\prime}, j^{\prime \prime}\right\rangle ; \sigma}\left[\hat{d}_{j^{\prime}, \sigma}^{L_{\mathbf{r}}}\right]^{\dagger} \hat{d}_{j^{\prime \prime}, \sigma}^{L_{\mathbf{r}}}$, where the hopping amplitude is $J_{L_{\mathrm{r}}}$ and $\hat{d}_{j, \sigma}^{L_{\mathrm{r}}}$ and $\left[\hat{d}_{j, \sigma}^{L_{\mathrm{r}}}\right]^{\dagger}$ are the fermionic operators in the rth lead. Finally the contact between the ferromagnetic system and its leads is described by $\mathcal{H}_{C}=$ $\sum_{\mathbf{r}} \mathcal{H}_{C}^{\mathrm{r}}$ with

$$
\mathcal{H}_{C}^{\mathbf{r}}=-J_{C}^{\mathbf{r}} \sum_{\sigma}\left[\hat{c}_{\mathbf{r}, \sigma}^{\dagger} \hat{d}_{\partial L_{\mathbf{r}}, \sigma}^{L_{\mathbf{r}}}+\left[\hat{d}_{\partial L_{\mathbf{r}}, \sigma}^{L_{\mathbf{r}}}\right]^{\dagger} \hat{c}_{\mathbf{r}, \sigma}\right]
$$

where $\partial L_{\mathbf{r}}$ denotes the last site of the lead and $J_{C}^{\mathbf{r}}$ is the amplitude of tunneling among both subsystems. An electric current flows through the FM by a difference in chemical potentials $\mu_{L}$ and $\mu_{R}$ that connect the left and right sides of the FM.

The spin current flowing from the system to the leads is determined as the rate of change of spin density in the rth site, i.e., $\frac{d \mathbf{s}^{\mathrm{r}}}{d t}=\frac{\hbar}{2 a^{2}} \frac{d}{d t} \sum_{\sigma, \sigma^{\prime}}\left\langle\hat{c}_{\mathbf{r}, \sigma}^{\dagger} \boldsymbol{\tau}_{\sigma, \sigma^{\prime}} \hat{c}_{\mathbf{r}, \sigma^{\prime}}\right\rangle$, where $a$ is the distance between sites. The general expression has been derived before ${ }^{28}$ and is given by

$$
\begin{aligned}
\frac{d \mathbf{s}^{\mathbf{r}}}{d t}= & \frac{i \hbar}{2 a^{2}} \int \frac{d \epsilon}{2 \pi} \operatorname{Tr}\left[N\left(\epsilon-\mu^{\mathbf{r}}\right) \Gamma_{\mathbf{r}, \mathbf{r}}^{\mathbf{r}}(\epsilon)\left(\boldsymbol{\tau} G_{\mathbf{r}, \mathbf{r}}^{R}(\epsilon)-G_{\mathbf{r}, \mathbf{r}}^{A}(\epsilon) \tau\right)\right. \\
& \left.-\sum_{\mathbf{r}^{\prime}} N\left(\epsilon-\mu^{\mathbf{r}^{\prime}}\right)\left(\boldsymbol{\tau} \Sigma_{\mathbf{r}, \mathbf{r}}^{\mathbf{r}, R}(\epsilon)-\Sigma_{\mathbf{r}, \mathbf{r}}^{\mathbf{r}, A}(\epsilon) \boldsymbol{\tau}\right) A_{\mathbf{r}, \mathbf{r}}^{\mathbf{r}^{\prime}}(\epsilon)\right],
\end{aligned}
$$

where $N(\epsilon)=\left[e^{\epsilon / k_{B} T}+1\right]^{-1}$ is the Fermi-Dirac distribution function with $k_{B} T$ the thermal energy, $A^{\mathbf{r}}(\epsilon)=$ $G^{R}(\epsilon) \hbar \Gamma^{\mathbf{r}}(\epsilon) G^{A}(\epsilon)$ is the spectral-weight contribution due to the lead at site $\mathbf{r}$ and the rate $\Gamma^{\mathbf{r}}(\epsilon)=i\left[\Sigma^{\mathbf{r}, R}(\epsilon)-\Sigma^{\mathbf{r}, A}(\epsilon)\right]$, where the superscript R (A) means the retarded (advanced) function (see Ref. 28 for details). Since we are assuming nonmagnetic leads, the self-energy $\Sigma^{\mathbf{r}, R}(\epsilon)$ will be proportional to the identity in spin space. Its only nonzero matrix elements are $\hbar \Sigma_{\mathbf{r}, \sigma ; \mathbf{r}, \sigma^{\prime}}^{\mathbf{r}, R}=-\left(J_{C}^{\mathbf{r}}\right)^{2} e^{i k^{\mathbf{r}}(\epsilon) a} \delta_{\sigma, \sigma^{\prime}} / J_{L_{\mathbf{r}}}$, with $k^{\mathbf{r}}(\epsilon) a=\arccos \left[-\epsilon / 2 J_{L_{\mathrm{r}}}\right]$. To carry out the explicit evaluation of Eq. (4) in terms of the magnetization orientation $\boldsymbol{m}_{\mathbf{r}}$ it is convenient to decompose the Green's functions into spinindependent singlet and spin-dependent triplet parts, namely

$$
G_{\mathbf{r}, \sigma ; \mathbf{r}^{\prime}, \sigma^{\prime}}^{R}(\epsilon)=G_{\mathbf{r}, \mathbf{r}^{\prime}}^{(s) R}(\epsilon) \delta_{\sigma, \sigma^{\prime}}+G_{\mathbf{r}, \mathbf{r}^{\prime}}^{(t) R}(\epsilon) \boldsymbol{m}_{\mathbf{r}} \cdot \boldsymbol{\tau}_{\sigma, \sigma^{\prime}},
$$

and likewise for the advanced Green's function. Taking the trace over the spin indices in Eq. (4) we distinguish two contributions to the spin current, one component parallel to the magnetization vector and other transverse, denoted by $\boldsymbol{j}_{s}^{\|}(\mathbf{r})$ and $\boldsymbol{j}_{s}^{\text {in }}(\mathbf{r})$, respectively. The transverse spin current density induced by the magnetic texture to lead $\mathbf{r}$ is given by

$$
\boldsymbol{j}_{s}^{\mathrm{in}}(\mathbf{r})=\frac{1}{a^{2}} \int \frac{d \epsilon}{(2 \pi)} \sum_{\mathbf{r}^{\prime}} N\left(\epsilon-\mu^{\mathbf{r}^{\prime}}\right) \mathbf{t}_{\mathbf{r r}^{\prime}}^{(t)}(\epsilon)\left(\boldsymbol{m}_{\mathbf{r}} \times \boldsymbol{m}_{\mathbf{r}^{\prime}}\right),
$$

with the transmission probability $\mathrm{t}_{\mathrm{rr}^{\prime}}^{(t)}(\epsilon)=$ $\hbar \Gamma_{\mathbf{r}, \mathbf{r}}^{\mathbf{r}}(\epsilon) G_{\mathbf{r}, \mathbf{r}^{\prime}}^{(t) R}(\epsilon) \hbar \Gamma_{\mathbf{r}^{\prime}, \mathbf{r}^{\prime}}^{\mathbf{r}^{\prime}}(\epsilon) G_{\mathbf{r}^{\prime}, \mathbf{r}}^{(t) A}(\epsilon)$ for the spin-polarized part of the current flowing from the lead at site $\mathbf{r}$ to the lead at site $\mathbf{r}^{\prime}$ through the FM. We now consider a zero net current (but nonzero spin current) flow into the leads, except for the left and right leads that have chemical potentials $\mu_{L}=\epsilon_{F}+|e| V$ and $\mu_{R}=\epsilon_{F}$, respectively, with $\epsilon_{F}$ the Fermi energy. At low temperatures and assuming the length scale of magnetization-orientation variation much greater than the inverse Fermi wavelength we see that in the continuum limit the only contributions to Eq. (6) are from neighboring leads for which $\boldsymbol{m}_{\mathbf{r}} \times \boldsymbol{m}_{\mathbf{r}^{\prime}} \rightarrow \boldsymbol{m}(\mathbf{x}) \times a \partial_{x} \boldsymbol{m}(\mathbf{x})$, taking the current in the $x$ direction. Keeping these contributions, we find that the transverse spin-current density to lowest order in magnetization gradients satisfies Eq. (1) with $g=a\left(16 M_{s} / g_{L} \mu_{B} P\right) \times$ $\left[G_{0} \mathrm{t}^{\mathrm{t}}\left(\epsilon_{F}\right) / \mathrm{t}\left(\epsilon_{F}\right)\right]$, where $\mathrm{t}\left(\epsilon_{F}\right)$ is the total (i.e., summed for both spin channels) transmission probability. Both this transmission probability, and the spin-dependent transmission probability are taken between leads at neighboring sites and are taken at the Fermi energy. Also note that these transmission probabilities are evaluated for the homogeneous ferromagnetic state, as Eq. (6) is already first order in magnetization gradient.

\section{SPIN SPIRAL AND INVERSE SPIN HALL EFFECT DETECTION}

We now calculate the spin accumulation in the normal metal due to the injected spin current, for a static magnetization texture $\boldsymbol{m}(x)$ depending on the coordinate $x$ along the wire. The spin diffusion equation for the spin accumulation is given by

$$
\nabla^{2} \boldsymbol{\mu}_{s}=\frac{\boldsymbol{\mu}_{s}}{\lambda_{\mathrm{sd}}^{2}},
$$

where $\lambda_{\mathrm{sd}} \equiv \sqrt{D_{s} \tau_{\mathrm{sf}}}$ is the spin-diffusion length in the NM, and $D_{s}$ and $\tau_{\text {sf }}$ are its spin-diffusion constant and spin-flip time, respectively. The boundary conditions for Eq. (7) enforce 
continuity for the spin current and are given by

$$
\begin{gathered}
\left.\partial_{z} \boldsymbol{\mu}_{s}(x, z)\right|_{z=0}=-\frac{G_{0}}{\sigma} j_{s, z}^{\text {net }}(x), \\
\left.\partial_{z} \boldsymbol{\mu}_{s}(x, z)\right|_{z=d_{N}}=0, \\
\left.\partial_{x} \boldsymbol{\mu}_{s}(x, z)\right|_{x= \pm L_{N}}=0,
\end{gathered}
$$

where $L_{N}, d_{N}$, and $\sigma$ are the length, thickness, and conductivity of the NM, respectively, and $j_{s, z}^{\text {net }}(x)$ is the net spin current into the NM. The net spin current is the sum of the spin injection, as given in Eq. (1), and a backflow spin current $\boldsymbol{j}_{s}^{\text {back }}$ in the opposite direction due to the induced spin accumulation on the NM side of the interface. Thus $\boldsymbol{j}_{s, z}^{\text {net }}=\boldsymbol{j}_{s, z}^{\text {in }}+\boldsymbol{j}_{s, z}^{\text {back }}$, where $^{29}$

$$
\boldsymbol{j}_{s, z}^{\mathrm{back}}=\frac{g^{\uparrow \downarrow}}{4 \pi G_{0}} \boldsymbol{\mu}_{s}(x, z=0),
$$

where $g^{\uparrow \downarrow}$ is the mixing conductance. (We neglect the imaginary part of the mixing conductance, which is justified for realistic interfaces. ${ }^{30}$ ) We note that in the absence of dissipation that leads to spin relaxation in the normal metal the net spin current across the interface would be zero as the injected spin current would be exactly canceled by the backflow spin current.

For a static helical, i.e., spiral magnetization, given by $\boldsymbol{m}_{\mathrm{sp}}(\boldsymbol{x})=(0, \cos q x, \sin q x)^{T}$, the injected tranverse spin current is independent of the $x$ coordinate, and can be measured using a Hall-type geometry possible as shown in Fig. 1. For an FM-Pt bilayer with this geometry the large ISHE $^{31,32}$ in the Pt converts the injected spin current into a voltage difference perpendicular to the direction of applied current. After solving the spin diffusion equation the voltage difference averaged over the thickness $d_{N}$ of the Pt layer due to the ISHE is given by

$$
\frac{V_{\mathrm{ISHE}}}{g / g \uparrow \downarrow}=\frac{\hbar W \theta_{\mathrm{SH}}}{d_{N}|e|} q v_{s} \frac{\cosh \frac{d_{N}}{\lambda_{\mathrm{sd}}}-1}{\frac{4 \pi \sigma}{g^{\uparrow \downarrow} \lambda_{\mathrm{sd}}} \sinh \frac{d_{N}}{\lambda_{\mathrm{sd}}}+\cosh \frac{d_{N}}{\lambda_{\mathrm{sd}}}},
$$

where $W$ is the width of the FM-Pt interface and $\theta_{\mathrm{SH}}$ is the spin Hall angle of Pt. Note that the thickness of the Pt-layer influences the signal considerably. ${ }^{33}$ For the ferromagnet we take the helimagnet MnSi which supports spin spirals with $q \sim 2 \pi / 20 \mathrm{~nm}^{-1}$. For a MnSi-Pt interface we estimate $g^{\uparrow \downarrow} \approx$ $10^{15} \Omega^{-1} \mathrm{~m}^{-2}, \sigma_{\mathrm{Pt}}=9.5 \times 10^{6} \Omega^{-1} \mathrm{~m}^{-1}, \lambda_{\mathrm{sd}}=1.5 \mathrm{~nm}$, and a platinum thickness of $d_{N}=3 \mathrm{~nm}, \theta_{\mathrm{SH}}=0.05$, and obtain $V_{\text {ISHE }} / W \approx 1.5\left(g / g^{\uparrow \downarrow}\right) \mathrm{V} / \mathrm{m}$, for a current density in the MnSi of $j_{c} \approx 10^{11} \mathrm{~A} \mathrm{~m}^{-2}$, where we assumed the spin velocity is of the order of the drift velocity.

\section{INTERFACIAL ENHANCEMENT OF SPIN TRANSFER}

Another implication of the injection of the spin current is the effect it has on the current-driven motion of, e.g., a domain wall. This is important for the understanding of domain-wall dynamics in layered magnetic materials, that are the subject of ongoing research. ${ }^{34}$ Note that up to this point we only considered static textures. For a moving domain wall we should however also include the spin-pumping contribution ${ }^{12,13}$

$$
\boldsymbol{j}_{s}^{\text {pump }}=\frac{\hbar g^{\uparrow \downarrow}}{4 \pi G_{0}} \boldsymbol{m} \times \frac{\partial \boldsymbol{m}}{\partial t} .
$$

For the inhomogeneous injection of transverse spin current the solution of Eq. (7) for the spin accumulation yields $\mu_{s}(x, z)=$ $\int d x^{\prime} K\left(x-x^{\prime}, z\right) j_{s, z}^{\text {net }}\left(x^{\prime}\right)$ where the net spin current is given by $\boldsymbol{j}_{s, z}^{\text {net }}=\boldsymbol{j}_{s, z}^{\text {in }+ \text { pump }}+\boldsymbol{j}_{s, z}^{\text {back }}$. The Fourier transform of the kernel $K(x, z)$ is given by

$\tilde{K}\left(k_{x}, z\right)=-\frac{G_{0} \lambda_{s d}}{\sigma} \frac{\cosh \left(\frac{z+d_{N}}{\lambda_{s d}} \sqrt{k_{x}^{2} \lambda_{s d}^{2}+1}\right)}{\sqrt{k_{x}^{2} \lambda_{s d}^{2}+1} \sinh \left(\frac{d_{N}}{\lambda_{s d}} \sqrt{k_{x}^{2} \lambda_{s d}^{2}+1}\right)}$.

The total spin current ejected from the FM layer reduces the angular momentum of the FM, inducing a torque which modifies the domain-wall dynamics. The Landau-LifshitzGilbert (LLG) equation in the presence of this additional torque is given by

$$
\begin{aligned}
\frac{\partial \boldsymbol{m}}{\partial t}+v_{s} \frac{\partial \boldsymbol{m}}{\partial x}= & \alpha_{0} \boldsymbol{m} \times \frac{\partial \boldsymbol{m}}{\partial t}-\frac{1}{\hbar} \boldsymbol{m} \times \frac{\delta E_{\mathrm{MM}}}{\delta \boldsymbol{m}} \\
& +\beta_{0} v_{s} \boldsymbol{m} \times \frac{\partial \boldsymbol{m}}{\partial x}+\frac{\gamma}{M_{s} d_{F}} j_{s, z}^{\text {net }},
\end{aligned}
$$

where $\alpha_{0}$ and $\beta_{0}$ are the bulk Gilbert damping and nonadiabaticity parameter, respectively, and $\gamma$ is the gyromagnetic ratio. Note that the spin current $\boldsymbol{j}_{\text {net }}$ now also includes the spin pumping contribution in Eq. (12) as we now consider a dynamic texture. The FM has thickness $d_{F}$ and hardaxis anisotropy $K_{\perp}$ which we take to be along the $y$ axis and is included in the energy functional $E_{\mathrm{MM}}$ that also contains the exchange and easy-axis anisotropy that set the domain-wall width. The domain wall is described by the collective coordinates $\varphi_{\mathrm{dw}}(t)$ which is the azimuthal angle and $r_{\mathrm{dw}}(t)$ which is the domain-wall position that enters in the polar angle $\theta_{\mathrm{dw}}(x, t)=2 \arctan \exp \left[x-r_{\mathrm{dw}}(t)\right] / \lambda_{\mathrm{dw}},{ }^{35}$ viz. $\boldsymbol{m}=\left(\cos \varphi_{\mathrm{dw}} \sin \theta_{\mathrm{dw}}, \sin \varphi_{\mathrm{dw}} \sin \theta_{\mathrm{dw}}, \cos \theta_{\mathrm{dw}}\right)^{T}$. From the LLG equation [Eq. (14)] we obtain the equations of motion for the collective coordinates of the domain wall, which are given by

$$
\begin{gathered}
\dot{\varphi}_{\mathrm{dw}}+\alpha_{\varphi} \frac{\dot{r}_{\mathrm{dw}}}{\lambda_{\mathrm{dw}}}=\beta_{\varphi} \frac{v_{s}}{\lambda_{\mathrm{dw}}}, \\
\frac{\dot{r}_{\mathrm{dw}}}{\lambda_{d w}}-\alpha_{r} \dot{\varphi}_{\mathrm{dw}}=\frac{K_{\perp}}{2 \hbar} \sin 2 \varphi_{\mathrm{dw}}+\frac{v_{s}}{\lambda_{\mathrm{dw}}},
\end{gathered}
$$

where $\alpha_{r, \varphi}$ and $\beta_{\varphi}$ are given by

$$
\begin{aligned}
\alpha_{r, \varphi} & =\alpha_{0}+\frac{\gamma \hbar g^{\uparrow \downarrow}}{4 \pi M_{s} d_{F}} \mathcal{I}_{r, \varphi}, \quad \beta_{\varphi}=\beta_{0}+\frac{\gamma \hbar g}{4 \pi M_{s} d_{F}} \mathcal{I}_{\varphi}, \\
\mathcal{I}_{\xi} & =\frac{4 \pi G_{0}}{\hbar g^{\uparrow \downarrow}} \iint d x d x^{\prime} \Pi\left(x-x^{\prime}\right) j_{s, z}^{\text {in }+ \text { pump }}(x) \cdot \frac{\delta \boldsymbol{m}_{\mathrm{dw}}}{\delta \xi}\left(x^{\prime}\right),
\end{aligned}
$$

with $\xi=\{r, \varphi\}$ and where $\Pi\left(x-x^{\prime}\right)$ is given by

$$
\Pi\left(x-x^{\prime}\right)=\int_{-\infty}^{\infty} \frac{d k_{x}}{2 \pi}\left[1-\frac{g^{\uparrow \downarrow}}{4 \pi G_{0}} \tilde{K}\left(k_{x}, 0\right)\right]^{-1} e^{i k_{x}\left(x-x^{\prime}\right)} .
$$

Note that the integrals $\mathcal{I}_{\varphi, r}$ are functions of the dimensionless parameters $\lambda_{\mathrm{dw}} / \lambda_{\mathrm{sd}}, d_{N} / \lambda_{\mathrm{sd}}$, and $g^{\uparrow \downarrow} \lambda_{\mathrm{sd}} / 4 \pi \sigma$. The average velocity of the current-driven domain wall is given by

$$
\left\langle\dot{r}_{\mathrm{dw}}\right\rangle=\frac{\beta_{\varphi}}{\alpha_{\varphi}} v_{s}+\frac{\operatorname{sign}\left[1-\frac{\beta_{\varphi}}{\alpha_{\varphi}}\right]}{1+\alpha_{r} \alpha_{\varphi}} \operatorname{Re}\left[\sqrt{\left(1-\frac{\beta_{\varphi}}{\alpha_{\varphi}}\right)^{2} v_{s}^{2}-v_{c}^{2}}\right],
$$




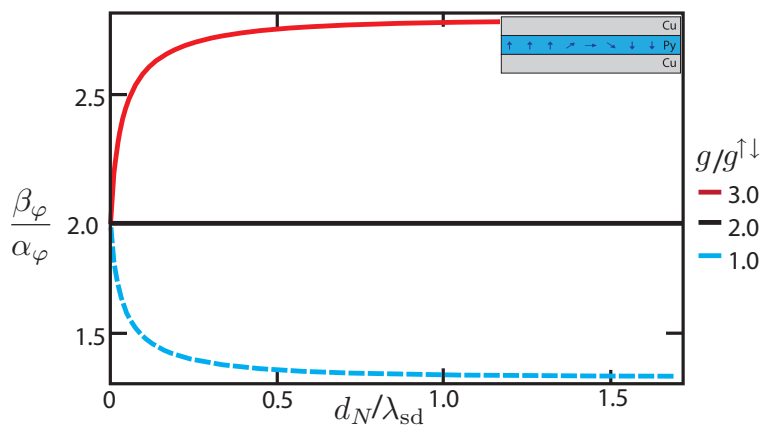

FIG. 2. (Color online) The ratio $\beta_{\varphi} / \alpha_{\varphi}$ is shown as a function of the thickness $d_{\mathrm{N}}$ of the normal-metal layers in units of its spin diffusion length $\lambda_{\mathrm{sd}}=250 \mathrm{~nm}$, for a Cu-Py-Cu multilayer. The headto-head domain wall in the Py has a width of $100 \mathrm{~nm}$. For $g / g^{\uparrow \downarrow}=2$, where $g^{\uparrow \downarrow}=1.6 \times 10^{15} \Omega^{-1} \mathrm{~m}^{-2}$ is the mixing conductance of a $\mathrm{Py}-\mathrm{Cu}$ interface, the ratio $\beta_{\varphi} / \alpha_{\varphi}=2$ coincides with the bulk ratio with $\alpha_{0}=0.006$.

where the critical velocity is given by $v_{c}=K_{\perp} \lambda_{\mathrm{dw}} / 2 \hbar$. The ratio $\beta_{\varphi} / \alpha_{\varphi}$ determines the qualitative behavior of the domainwall velocity as a function of current. In Fig. 2 this ratio is shown as a function of the thickness of the normal-metal layers in the multilayer. Note that for $d_{N} \gg d_{F}$ the interface enhancement dominates over the bulk values, and that in the limit of a large ratio $\lambda_{\mathrm{dw}} / \lambda_{\text {sd }}$ our result for $\alpha_{\varphi}$ coincides with interfacial enhancement of Gilbert damping in singledomain magnets. ${ }^{12,13}$ Also note that for moving domain walls $j_{s, z}^{\text {in }+ \text { pump }} \propto\left(g v_{s}-g^{\uparrow \downarrow} v_{\mathrm{dw}}\right)$, so that the net injected spin current goes down once the domain wall is set into motion.

\section{DISCUSSION AND CONCLUSIONS}

In this article we describe a mechanism for transverse spin injection across a FM-NM interface that is induced by a magnetization gradient along the current, and focus on the examples of spin spirals and domain walls. In the example of a domain wall, the spin-current injection takes place at the position of the domain wall which could lead to spin injection with control over spin polarization and location of injection. Moreover, due to the spin accumulation induced in the NM layer(s) the measured $\alpha$ and $\beta$ parameters for domain-wall motion in multilayer systems will be different than their bulk values. We expect that this effect plays a role in all metallic heterostructure thin-film measurements of domain-wall dynamics. In fact, large values of beta are typically reported in such systems, ${ }^{36}$ pointing to the possibility of interfacial enhancement.

We can make a connection between the transverse spincurrent injection and spin pumping. Consider spin pumping by a precessing spiral magnetization in the absence of a current [see Eq. (1)], when the wavelength of the helix is much larger than the spin diffusion length in the ferromagnet. If the system were Galilean invariant a Galilean transformation to the frame moving with velocity $\omega / q$ would yield a static magnetization and a nonzero spin current leading to transverse spin injection as given by Eq. (1) with $g / g^{\uparrow \downarrow}=1$. Therefore we expect $g / g^{\uparrow \downarrow}$ to be of order unity. However, since Galilean invariance in practice is broken there is a difference between injection and pumping, analogous to the difference between the $\alpha$ and $\beta$ parameters in the LLG equation. This is also reflected in the fact that the parameter $g$ is a three-terminal transport property, since it involves transverse spin transport into one lead in response to longitudinal charge transport driven through two other leads [see Eq. (6)], which is microscopically distinct from the two-terminal scattering processes contributing to $g^{\uparrow \downarrow}$ and spin pumping.

It is also possible to give another interpretation of magnetictexture-controlled transverse spin injection. We do this by considering the adiabatic spin transfer torque (STT) ${ }^{37,38}$

$$
\left.\frac{\partial \boldsymbol{m}}{\partial t}\right|_{\mathrm{STT}}=-\left(\boldsymbol{v}_{s} \cdot \nabla\right) \boldsymbol{m} \propto \boldsymbol{m} \times\langle\boldsymbol{s}\rangle,
$$

and interpret it as a torque due to a current-induced spin density $\langle\boldsymbol{s}\rangle \propto \boldsymbol{m} \times\left(\boldsymbol{v}_{s} \cdot \nabla\right) \boldsymbol{m}$ of the itinerant electrons. This nonequilibrium spin density may leak into the normal metal, giving rise to a spin current of the form in Eq. (1). We stress again, however, that for a microscopic treatment one has to resort to the formalism of Sec. II as, e.g., a diffusive treatment of the nonequilibrium spin density $\langle s\rangle$ with boundary conditions at the interface between FM and NM will not involve the parameter $g$ and thus not account correctly for the lack of Galilean invariance.

We also note that in principle there is a spin current with spin polarization in the direction of $\left(\boldsymbol{v}_{s} \cdot \boldsymbol{\nabla}\right) \boldsymbol{m}$ that we have ignored as it oscillates and averages out to small values when integrated over position, and is determined approximately by the imaginary part of the mixing conductance which is small for realistic interfaces.

Other spin-injection mechanisms, such as the spindependent Seebeck effect ${ }^{25}$ or diffusive spin injection, ${ }^{17}$ typically induce a spin current in the NM layer with the spin polarization parallel to the magnetization in the ferromagnet. Therefore the spin injection we discussed in this article, which induces a spin current polarized in the direction of the helicity of the magnetic texture along the current, is distinguishable even when an inhomogeneous current distribution leads to current paths through the FM-NM interface and thus to injection of spin with polarization along the magnetization. Moreover, the spin current we propose is tunable in position and polarization via manipulation of the magnetization, in contrast to the SHE and other mechanisms of spin injection.

The Onsager reciprocal process of the spin-injection mechanism we discussed here occurs as well. A spin current with a spin direction aligned to the helicity of a magnetization texture injected into a ferromagnet leads to a voltage difference over the ferromagnetic strip. In this way domain walls could be used as a local movable sensors of spin current. In future work we plan to investigate transverse pure spin currents associated with heat currents in the same geometry that is discussed here.

\section{ACKNOWLEDGMENTS}

It is a pleasure to thank Gerrit Bauer, Gavin Burnell, Bryan Hickey, Mathias Kläui, Chris Marrows, Tom Moore, and Maxim Tsoi for discussions. This work was supported by the Stichting voor Fundamenteel Onderzoek der Materie (FOM), the Netherlands Organization for Scientifc Research (NWO), and the European Research Council (ERC). 
*e.vanderbijl@uu.nl

${ }^{1}$ R. Jansen, Nat. Mater. 11, 400 (2012).

${ }^{2}$ S. A. Wolf, D. D. Awschalom, R. A. Buhrman, J. M. Daughton, S. von Molnr, M. L. Roukes, A. Y. Chtchelkanova, and D. M. Treger, Science 294, 1488 (2001).

${ }^{3}$ S. Takahashi and S. Maekawa, Sci. Technol. Adv. Mater. 9, 014105 (2008); I. Žutić, J. Fabian, and S. Das Sarma, Rev. Mod. Phys. 76, 323 (2004).

${ }^{4}$ P. W. Brouwer, Phys. Rev. B 58, R10135 (1998).

${ }^{5}$ G. Lampel, Phys. Rev. Lett. 20, 491 (1968).

${ }^{6}$ S. D. Ganichev, E. L. Ivchenko, S. N. Danilov, J. Eroms, W. Wegscheider, D. Weiss, and W. Prettl, Phys. Rev. Lett. 86, 4358 (2001).

${ }^{7}$ M. J. Stevens, A. L. Smirl, R. D. R. Bhat, A. Najmaie, J. E. Sipe, and H. M. van Driel, Phys. Rev. Lett. 90, 136603 (2003).

${ }^{8}$ C. W. Sandweg, Y. Kajiwara, A. V. Chumak, A. A. Serga, V. I. Vasyuchka, M. B. Jungfleisch, E. Saitoh, and B. Hillebrands, Phys. Rev. Lett. 106, 216601 (2011)

${ }^{9}$ K. Ando, S. Takahashi, J. Ieda, H. Kurebayashi, T. Trypiniotis, C. Barnes, S. Maekawa, and E. Saitoh, Nat. Mater. 10, 655 (2011).

${ }^{10}$ H. Kurebayashi, O. Dzyapko, V. Demidov, D. Fang, A. Ferguson, and S. Demokritov, Nat. Mater. 10, 660 (2011).

${ }^{11}$ M. Weiler, H. Huebl, F. S. Goerg, F. D. Czeschka, R. Gross, and S. T. B. Goennenwein, Phys. Rev. Lett. 108, 176601 (2012).

${ }^{12}$ Y. Tserkovnyak, A. Brataas, and G. E. W. Bauer, Phys. Rev. Lett. 88, 117601 (2002).

${ }^{13}$ Y. Tserkovnyak, A. Brataas, and G. E. W. Bauer, Phys. Rev. B 66, 224403 (2002).

${ }^{14}$ S. Mizukami, Y. Ando, and T. Miyazaki, Phys. Rev. B 66, 104413 (2002).

${ }^{15}$ E. Shikoh, K. Ando, K. Kubo, E. Saitoh, T. Shinjo, and M. Shiraishi, Phys. Rev. Lett. 110, 127201 (2013).

${ }^{16}$ M. Johnson and R. H. Silsbee, Phys. Rev. B 35, 4959 (1987); 37, 5312 (1988).

${ }^{17}$ F. J. Jedema, M. S. Nijboer, A. T. Filip, and B. J. van Wees, Phys. Rev. B 67, 085319 (2003).

${ }^{18}$ P. C. van Son, H. van Kempen, and P. Wyder, Phys. Rev. Lett. 58, 2271 (1987)

${ }^{19}$ S. Dash, S. Sharma, R. Patel, M. De Jong, and R. Jansen, Nature (London) 462, 491 (2009).

${ }^{20}$ M. I. D'Yakonov and V. I. Perel', JETP Lett. 13, 467 (1971); M. Dyakonov and V. Perel, Phys. Lett. A 35, 459 (1971); J. E. Hirsch, Phys. Rev. Lett. 83, 1834 (1999).

${ }^{21}$ V. Sih, W. H. Lau, R. C. Myers, V. R. Horowitz, A. C. Gossard, and D. D. Awschalom, Phys. Rev. Lett. 97, 096605 (2006).

${ }^{22}$ S. Murakami, N. Nagaosa, and S.-C. Zhang, Science 301, 1348 (2003).

${ }^{23}$ J. Sinova, D. Culcer, Q. Niu, N. A. Sinitsyn, T. Jungwirth, and A. H. MacDonald, Phys. Rev. Lett. 92, 126603 (2004).

${ }^{24}$ K. Uchida, S. Takahashi, K. Harii, J. Ieda, W. Koshibae, K. Ando, S. Maekawa, and E. Saitoh, Nature (London) 455, 778 (2008).

${ }^{25}$ A. Slachter, F. Bakker, J.-P. Adam, and B. Van Wees, Nat. Phys. 6, 879 (2010).

${ }^{26}$ J.-C. Le Breton, S. Sharma, H. Saito, S. Yuasa, and R. Jansen, Nature (London) 475, 82 (2011).

${ }^{27}$ M. König, S. Wiedmann, C. Brüne, A. Roth, H. Buhmann, L. W. Molenkamp, X.-L. Qi, and S.-C. Zhang, Science 318, 766 (2007); C. L. Kane and E. J. Mele, Phys. Rev. Lett. 95, 146802 (2005).

${ }^{28}$ R. A. Duine, P. M. Haney, A. S. Núñez, and A. H. MacDonald, Phys. Rev. B 75, 014433 (2007).

${ }^{29}$ A. Brataas, Y. Nazarov, and G. Bauer, Eur. Phys. J. B 22, 99 (2001).

${ }^{30}$ K. Xia, P. J. Kelly, G. E. W. Bauer, A. Brataas, and I. Turek, Phys. Rev. B 65, 220401 (2002).

${ }^{31}$ E. Saitoh, M. Ueda, H. Miyajima, and G. Tatara, Appl. Phys. Lett. 88, 182509 (2006)

${ }^{32}$ T. Kimura, Y. Otani, T. Sato, S. Takahashi, and S. Maekawa, Phys. Rev. Lett. 98, 156601 (2007).

${ }^{33}$ V. Castel, N. Vlietstra, J. B. Youssef, and B. J. van Wees, Appl. Phys. Lett. 101, 132414 (2012).

${ }^{34}$ M. Hayashi, L. Thomas, C. Rettner, R. Moriya, Y. B. Bazaliy, and S. S. P. Parkin, Phys. Rev. Lett. 98, 037204 (2007).

${ }^{35}$ G. Tatara and H. Kohno, Phys. Rev. Lett. 92, 086601 (2004).

${ }^{36}$ I. M. Miron, P.-J. Zermatten, G. Gaudin, S. Auffret, B. Rodmacq, and A. Schuhl, Phys. Rev. Lett. 102, 137202 (2009).

${ }^{37}$ L. Berger, Phys. Rev. B 54, 9353 (1996).

${ }^{38}$ J. Slonczewski, J. Magn. Magn. Mater. 159, L1 (1996). 